\title{
Mehr als das Salz in der Suppe
}

\author{
Die Schweiz hängt beim Thema Versorgungsforschung ziemlich hinterher. Auf dem \\ ersten Schweizer Kongress zu diesem Thema diskutierten kürzlich engagiert Exper- \\ ten aus Medizin, Politik und Gesellschaft und kamen zu dem Schluss: Versorgungs- \\ forschung geht uns alle an.
}

\section{Felicitas Witte}

Fotos: Peter Brandenberger

* 1. Forum für Versorgungsforschung, Zürich 8.-9.5.2014. www.medicongress.ch/ kongresse/2014/Rueckblicke/ Versorgungsforschung14.php

Korrespondenz:

Dr. med. Felicitas Witte

Seefeldstrasse 285

CH-8008 Zürich

felicitas.witte[at]icloud.com

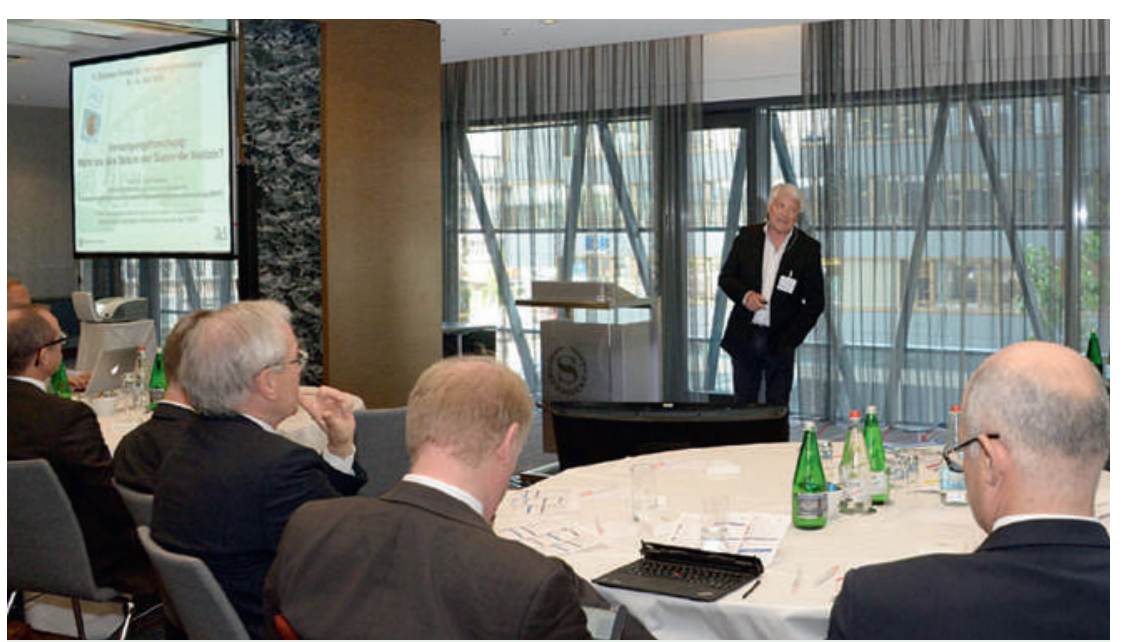

Gerd Glaeske, Hauptgeschäftsführer im Deutschen Netzwerk für Versorgungsforschung sieht Versorgungsforschung als gute Strategie, um Qualität und Effizienz zu verbessern, damit Patienten weder über-, unter- oder fehlversorgt werden.

Stellen Sie sich vor, eine Pharmafirma entwickelt ein neues Medikament gegen Diabetes. Die Studien sind überzeugend: Das Medikament vermeidet DiabetesKomplikationen viel besser als herkömmliche Präparate, und die Nebenwirkungen sind erträglich. Die Swissmedic lässt das Präparat zu, Tausende von Diabetikern erhalten das neue Medikament, und Versicherungen zahlen zähneknirschend den höheren Preis. Doch Jahre später finden Forscher heraus: Das Medikament wirkt gar nicht besser als die herkömmlichen, preiswerteren. Tausende von Franken wurden unnötig ausgegeben.

«Um so etwas zu vermeiden, brauchen wir Versorgungsforschung», sagt Willy Oggier, Gesundheitsökonom in Küsnacht. Versorgungsforschung bedeutet, vereinfacht gesagt, medizinische Behandlungen auf ihre Alltagstauglichkeit hin wissenschaftlich zu untersuchen. «In der Schweiz sind wir auf dem Gebiet nicht gerade Weltspitze», so Oggier. «Dabei ist das ein wichtiges Thema und wird im Zusammenhang mit DRG immer mehr an Gewicht gewinnen.» Der Gesundheitsökonom initiierte gemeinsam mit Doris Brandenberger, Geschäftsführerin von MediCongress, das erste Forum für Versorgungsforschung in der Schweiz, das im Mai in Zürich stattfand.* Dankbar sind die beiden für die Unterstützung des Zürcher Gesundheitsdirektors, Regierungsrat Thomas 


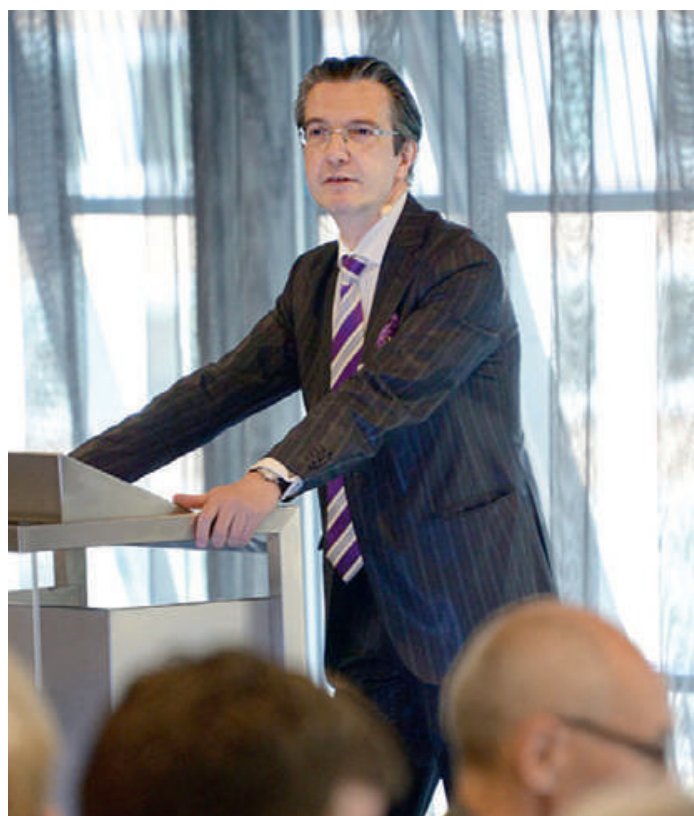

Willy Oggier: «Versorgungsforschung ist aufdeckende Forschung, und Transparenz gefällt nicht jedem.»

schliessen», forderte Glaeske. Bei vielen Medikamenten ist nicht belegt, dass sie Patienten einen Zusatznutzen bringen. So analysierte Mariam Ujeyl von der Arzneimittelkommission der Deutschen Ärzteschaft vor zwei Jahren 81 Zulassungsstudien für 39 Medikamente, die der Europäischen Zulassungsbehörde EMA eingereicht wurden. Bei nur 46 Prozent der Medikamente wurde ein patientenrelevanter Endpunkt untersucht und in nur 28 Prozent gab es genügend Daten, damit die EMA prüfen konnte, ob das neue Medikament einem herkömmlichen überlegen war. «Eine positive Zulassung bedeutet noch lange nicht, dass ein Medikament im Alltag auch wirklich nützt», erklärte Glaeske. «Deshalb brauchen wir qualitativ gute Versorgungsforschungs-Studien mit patientenrelevanten Endpunkten.»

\section{Versorgungsforschung als Chance sehen}

39 Prozent der Ärzte, so zeigte jedoch eine Studie von Klaus Lieb und Sabine Brandtönies von der Klinik für Psychiatrie und Psychotherapie an der Universitätsmedizin Mainz aus dem Jahre 2010, verordnen jedoch gerne neue Medikamente, weil sie ihren Patienten die neuesten Therapiemöglichkeiten anbieten und suggerieren möchten, sie seien stets auf dem neuesten wissenschaftlichen Stand. Transparenz und Aufklärung mit klaren Daten kann hier viel nützen, wie Glaeske anhand anschaulicher Beispiele aus der Praxis berichtete. So zeigte beispielsweise 2009 eine Studie im British Medical Journal, dass Antibiotika bei Kindern mit akuter Otitis media nicht wirken und sogar kontraindiziert sind: Kinder mit Amoxicillin bekamen nach 3,5 Jahren häufiger eine erneute Otitis. «Die Studie war ein wichtiges Argument dafür, Antibiotika bei den Kindern zurückhaltender zu verschreiben», sagt Glaeske. Die neuen
Erkenntnisse müssten natürlich mit entsprechenden Kampagnen weitergegeben werden: Etwa Informationsveranstaltungen der Krankenkassen für Versicherte oder thematisch orientierte Qualitätszirkel für Ärzte. «Doch auch wenn wir neue Studien zum Nutzen haben, kommt das leider bei vielen nicht an.» So zog die amerikanische Zulassungsbehörde FDA 2010 die Zulassung für das neue Brustkrebsmedikament Bevacizumab zurück. Bevacizumab verlängerte noch nicht einmal das Gesamtüberleben und erhöhte das Risiko für schwere unerwünschte Wirkungen um 20 Prozent. «Trotzdem verabreichen das in Deutschland immer noch einige Ärzte und der Hersteller bewirbt es», schimpfte Glaeske. Gefährlich findet der Gesundheitspolitiker auch den Trend, dass immer mehr Ärzte Kindern mit ADHS Neuroleptika verschreiben. «Vermutlich machen sie das, weil Ritalin in Verruf gekommen ist. Aber für den Nutzen von Neuroleptika gibt es überhaupt keine Daten.» Nein, er wolle keinen Pessimismus verbreiten. «Wir brauchen aber Versorgungsforschung, um all solche Probleme aufzudecken und anzugehen. Das geht nur mit Institutionen, die Zugang zu den entsprechenden Daten haben. Denn nur mit Daten können Taten folgen, das heisst evidenzbasierte Entscheidungen.»

Ärzte kamen in den Beispielen von Glaeske oftmals schlecht weg. Aus Angst, ihr unwissenschaftliches Verhalten könne aufgedeckt werden, würden manche Mediziner sicherlich die Augen zumachen vor dem Thema Versorgungsforschung. Einige Vorträge beschäftigten sich daher eingehend mit den Konsequenzen, die diese Forschung auf den Alltag von Medizinern hat. «Ärzte dürfen Versorgungsforschung nicht als Gefahr sehen, sondern als Chance», sagte Holger Pfaff, stellvertretender Vorsitzender des DNVF. Der Arztberuf unterliege einem grundlegenden Wandel, und Versorgungsforschung sei Teil dieses Wandels, den Mediziner nicht ignorieren dürften. Viele Patienten informieren sich heutzutage ausführlich im Internet über ihre Krankheit, lesen Leitlinien und sogar wissenschaftliche Studien. «Ärzte müssen heute mehr und mehr begründen, warum sie eine Therapie wählen oder von einer Leitlinie abweichen», sagte Pfaff. Der Patient der Zukunft nehme seine eigene Therapie eigenverant-

\section{Was ist Versorgungsforschung?}

Versorgungsforschung ist: «eine grundlagenund problemorientierte fachübergreifende Forschung, welche die Kranken- und Gesundheitsversorgung in ihren Rahmenbedingungen beschreibt, kausal erklärt und aufbauend darauf Versorgungskonzepte entwickelt, deren Umsetzung begleitend erforscht und/oder unter Alltagsbedingungen evaluiert».

Aus: Pfaff H. Versorgungsforschung: Begriffsbestimmung, Gegenstand und Aufgaben. In: Pfaff H, Schrappe M, Lauterbach KW, Engelmann U, Halber M (Hrsg.). Gesundheitsversorgung und Disease Management: Grundlagen und Anwendungen der Versorgungsforschung. Bern: Huber; 2003. 
wortlich mit in die Hand. «Versorgungsforschung entzaubert zwar den Arzt als «allmächtigen Halbgott in Weiss), aber es stärkt die Patientenautonomie», sagte Pfaff. «Mediziner sollten es sich als Ziel setzen, mit Patienten auf einer Augenhöhe zu reden und zu erklären, warum sie welche Behandlung vorschlagen würden. Dafür muss man natürlich wissen, welche Therapie dem Patienten nützt - und das geht nur mit Versorgungsforschung. Ärzte sollten es nicht als Gefahr sehen, sondern als Chance.»

Für die frei praktizierende Ärzteschaft sei Versorgungsforschung ein wichtiges Thema, sagte Peter Wiedersheim, Co-Präsident der Konferenz Kantonaler Ärztegesellschaften. So lösen Hausärzte rund 70 Prozent der Gesundheitsprobleme ihrer Patienten mit nur vier Prozent der direkt verursachten Gesundheitsausgaben, zitierte er eine Studie von Peter Tschudi vom Institut für Hausarztmedizin. «Hausärzte sind das Fundament der Medizin», so Wiedersheim, «und auch sie spielen eine wichtige Rolle bei der Versorgungsforschung.» Diese findet auf verschiedenen Ebenen statt. Auf regionaler, nationaler oder internationaler «Makro-Ebene» analysiert Versorgungsforschung das Gesundheitssystem «im Überblick» und dessen gesundheitspolitische, gesellschaftliche und volkswirtschaftliche Vorgaben. Auf der «Meso-Ebene» geht es darum, wie medizinische Leistungen oder Produkte unter Alltagsbedingungen, also in Spitälern oder Praxen, umgesetzt werden und wie effektiv sie sind. Die «Mikro-Ebene» untersucht schliesslich die individuelle Versorgungssituation, also die Beziehung zwischen Patient und Arzt. Die Versorgungsforschung freipraktizierender Ärzte finde zwar auf der Mikro- oder Meso-Ebene statt, erklärte Wiedersheim, und zwar vor allem im ambulanten Bereich. «Sie ist aber entscheidend für die Makro-Ebene, das heisst sie ist medizinisch und ökonomisch von grosser Bedeutung. Das wurde bisher sträflich vernachlässigt!» $\mathrm{Zu}$ wenig sei die Arbeit der freipraktizierenden Ärztinnen und Ärzte honoriert worden.

Dass die Versorgungsforschung in der Schweiz gestärkt werden müsse, ist für Christoph Bosshard, Mitglied des Zentralvorstandes der FMH, unbestritten. "Wir müssen evidenzbasierte Grundlagen für ein nachhaltiges und zukunftsorientiertes Gesundheitswesen schaffen. Dafür müssen wir mehr Experten ausbilden, die sich im Bereich der Versorgungsforschung auskennen. Wir müssen uns national und international besser vernetzen und später die Resultate verständlich kommunizieren.» Die FMH unterstützt seit 2011 zusammen mit der Konferenz der Kantonalen Ärztegesellschaften und NewIndex den Schwerpunkt Versorgungsforschung am Institut für Sozial- und Präventivmedizin (ISPM) in Bern. Eine weitere Finanzierung ist bis 2016 vorgesehen. Regelmässig tauscht sich die FMH mit den Forschern vom ISPM aus, ohne jedoch auf deren wissenschaftliche Arbeit Einfluss nehmen zu wollen. «Uns ist es ein grosses Anliegen, dass wir die Ärzte dafür sensibili-

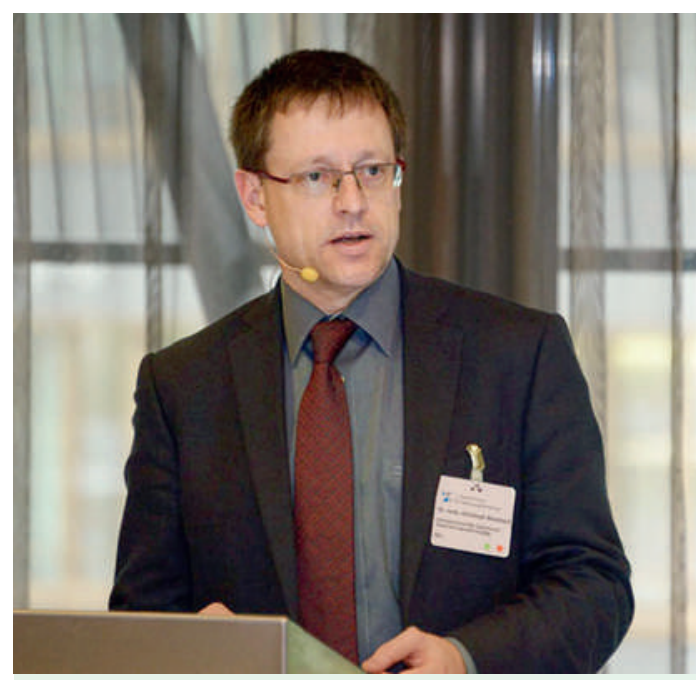

Christoph Bosshard: «Der FMH ist es ein grosses Anliegen, dass wir die Ärzte dafür sensibilisieren, wie wichtig Versorgungsforschung ist und dass sie es akzeptieren.»

sieren, wie wichtig Versorgungsforschung ist und dass sie es akzeptieren», sagte Bosshard. Noch seien einige Herausforderungen zu meistern. So ginge es nicht nach dem Motto one size fits all, sondern Patienten hätten unterschiedliche Bedürfnisse, Präferenzen und Perspektiven. «Diese Heterogenität muss Versorgungsforschung berücksichtigen», forderte Bosshard. So müsse es zum Beispiel in begründeten Fällen dem Arzt erlaubt sein, von Leitlinien abzuweichen. Zum Beispiel wenn diese sich widersprächen und der Arzt aus übergeordnet ganzheitlicher Sicht eine Priorität setze, weil er den Patienten gut kenne.

Ergebnisse der Versorgungsforschung würden aber nicht nur dem Patienten zugute kommen, sondern könnten auch politische Entscheidungen massgeblich beeinflussen, sagte Ignazio Cassis, Nationalrat der FDP im Tessin. «Uns fehlt vor allem auf Systemebene Versorgungsforschung. Ohne eine solche gibt es aber keine echten Innovationen. Da haben wir wirklich Nachholbedarf.» Während es zum Beispiel im Bereich der Grundlagen- und der klinischen Forschung ständig neue Methoden, Techniken oder Therapien gäbe, hinke man bei der Versorgungsforschung hinterher. «Zum einen gibt es bei der relativ jungen Disziplin noch zu wenig Erfahrung, zum anderen steckt die Methodologie der Versorgungsforschung noch in den Kinderschuhen», sagte Cassis. Ausserdem mangele es an finanziellen Mitteln. «Ein weiteres Problem ist, dass sich viele Disziplinen um Versorgungsforschung kümmern. Multidisziplinarität kann ein Reichtum sein, aber auch ein Hindernis. Epidemiologen ticken zum Beispiel ganz anders als Politiker.» Versorgungsforschung gehört zu den Prioritäten der Politik, versicherte Cassis. «Es ist in aller Munde, es ist in». Ich hoffe, das bleibt kein reines Modephänomen.»

Der nächste Kongress zur Versorgungsforschung soll am 18. und 19. Mai 2015 stattfinden. 